\title{
INTELLIGENT INTEGRATIVE MICRO-NANO-ROBOTICS
}

\author{
GHEORGHE, I[on] G[heorghe]; CIRSTOIU, C[armen] A[driana]; ISTRITEANU, S[imona] - E[lena] \& \\ DESPA, V[eronica]
}

\begin{abstract}
The scientific paper treats in original concept "inteligent integrative micro-nano-robotics" with the structure, principles and its special applications. In the context of the paper are presented the concepts of micro-nano robotic systems and new possibilities in micro-nano special applications processing and micro-nano technologies for layer on layer asssembly, with monitoring of structures topography on atomic force microscope, are presented also the concepts of measuring systems and digital control including the schemes for signal command and control for DAQ interface. The aim of this scientific paper is to develop new special applications in intelligent integrative micro-nano robotics domain, and in diagnosis medicine and treatment with experimentation / testing in situ.

Key words: micro-nano-robotics, micro-nano-manipulation, micro-nano-assembly, micro-nano-patterning, micro-nanoinstrumentation
\end{abstract}

\section{INTRODUCTION}

Micro-Nano-Robotics, is the engineering/advanced micronano-engineering dealing with the study, design, development and implementation of micrometric and nano-metric scale robotics for manufacturing micro and nano processes, micro and nano-manipulation and maintenance of micro-nanomeasurement and positioning for processes of micro and nanometrology, micro and nano-calibration and micro and nanocalibration for objects and landmarks for sub-systems, products and systems, which have dimensions of millimeters, micrometers and nanometers with high or ultra-precise resolution.

\section{CONCEPTS, STRUCTURES \& APPLICATIONS OF MICRO-NANO-INTELLIGENT ROBOTICS}

\subsection{The Intelligent Micro-Nano-Robotics Concept}

The Intelligent Micro-Nano-Robotics Concept (Gheorghe, 2009) is a new integrative intelligent science and synergistic and generative concept that deals with the study of robotics at "micro" and "nano" scale and includes robots that are of micronano size and are able to manipulate objects with nanometer dimensions of the order and micro-nanometer and micronanometer resolution.

\subsection{Structures and Principles of Micro-Nano-Intelligent Robotics}

The new structures and principles (Gheorghe, 2009) address the Micro-Nano-intelligent robot, define the development of hardware and software of the world "micro nano" world, approaching information processing and the intelligent manufacturing process.

In this context, the authors have conceived and designed an integrated micro-nano-system shown in Figure 1, a new technical and technological concept and a hybrid and hyperintegrated solution.
By using and combining processes such as "tip-base" and "base-tip", you can create a new hybrid micro-nano-robotics (Fig. 2), which is based on micro-nano-robotics and micronano-manipulation and a new technique for structuring nanomaterials and nano-structures as a new technique of nanofabrication.

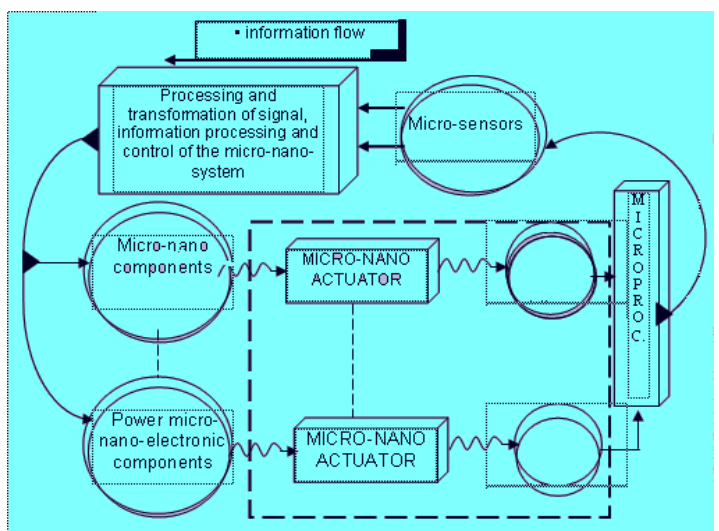

Fig. 1. Concept for micro-nano systems

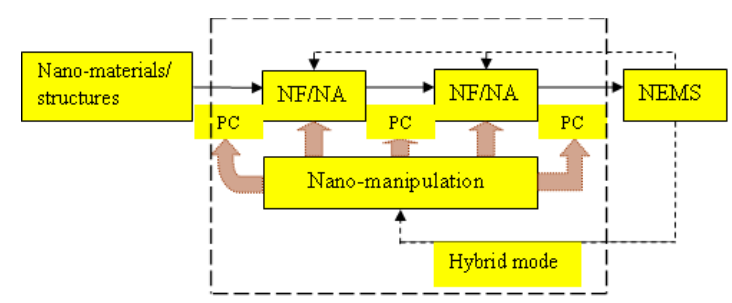

Fig. 2. Nano-materials nano-manipulation

Other applications of Intelligent Micro-Nano-Robotics are:

- Micro-nano-processing:

- Micro-nano-structuring:

○ Engineering / technology layer on layer assembly (Fig. 3 based on NAM - VA / 10 B played by AFM topography).

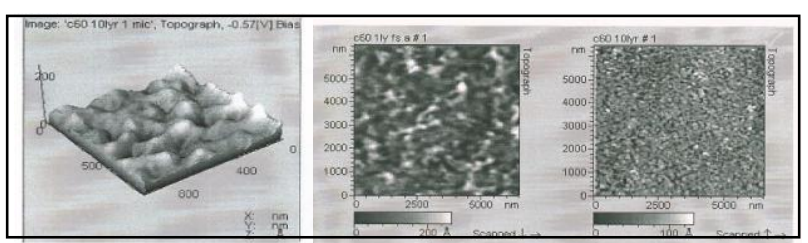

Fig. 3. Technology layer on layer based on AFM topography

\section{CONCEPTUAL AND CONSTRUCTIVE DEVELOPMENT OF INTELLIGENT MICRO- NANO-ROBOTICS}

The conceptual and constructive development of "Intelligent Micro-Nano-Robotics" includes Virtual MicroNano-Robotics, generated in an advanced hardware and software combination. 
Virtual Micro-Nano-Robotics requires modular, intelligent and hyper-integrated parts, especially intelligent and hyperintelligent software.

Virtual Micro-Nano-Robotics is used especially in the Micro-Nano-Robotics of Intelligent Measurement, where the measurement is to represent the relations between physical quantities and other sizes available directly on the micro-nanorobotics terminal, and allows measurements of physical sizes using electrical quantities (voltage, current intensity, etc.) so that all physical quantities will be converted into electrical signals.

The digital measuring signals from the micro-nano-digital measurement systems, based on the facility to transmit information as a binary code, a code that is interpreted by the micro-nano-devices and turned into useful information numeric and / or graphic display (Fig. 4).

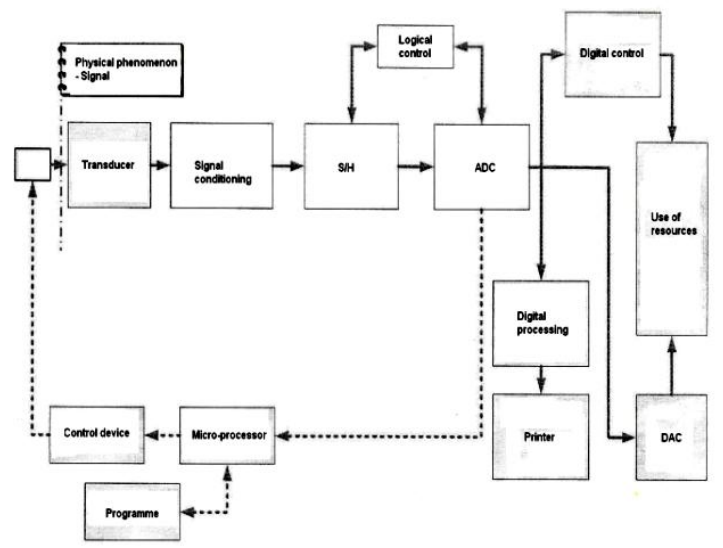

Fig. 4. Components of a measurement system / digital control

In Fig. 5 is shown, the principle diagram for control signals and control interface card type data acquisition (DAQ)

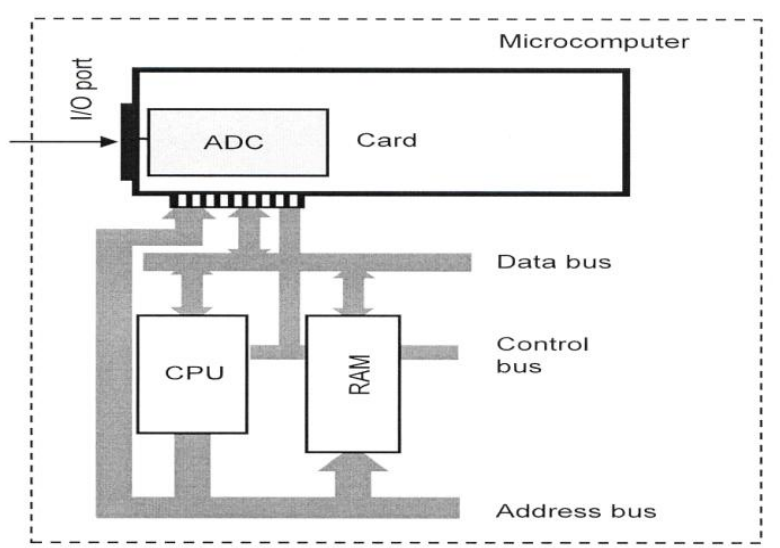

Fig. 5. The principle scheme for command and control signals for an interface type data acquisition card (DAQ)

\section{SPECIAL APPLICATIONS OF MICRO-NANO- ROBOTICS}

Special Applications of Micro-Nano-Robotics Intelligent found in:

- In-situ process where nano-robots are introduced into the human body to detect a tissue or cell of interest, followed by positioning and securing the area or the surrounding part;

- The process of investigation where nano-robots are placed in the human body in areas of interest and determine the parameters and record parameters or values to be communicated to the environment that makes the investigation for making a correct diagnosis;
Next, the area is rendered the area of intelligent MicroNano-Robotics applications (Fig. 6) in other media, such as:

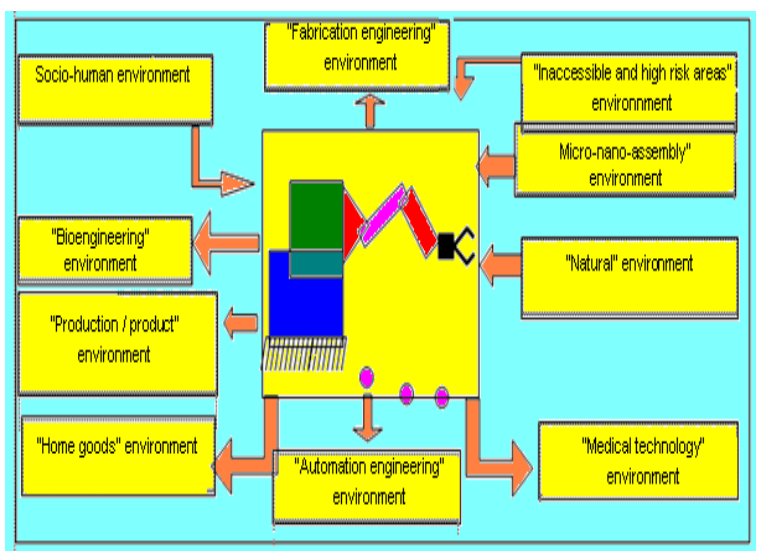

Fig. 6. Area of intelligent Micro-Nano-Robotics applications

\section{RESEARCH CONTRIBUTION PERSPECTIVES}

The hyphotesis and the target of realised researches were materialised in elaborating contributions scientifically fundamented by the new concepts regarding intelligent integrative micro-nanorobotics and micro systems for measuring and digital control and the schemes for command and signal control for interface. Also, realised researches were materialised in experimentation / testing of laborator applications and in special applications regarding micro-nano technologies layer on layer assembly based on AFM topography and regarding the procedures and diagnostic and treatment techniques for in situ processes. The research directions for perspective based on presented researches should be vectorized, step by step, according to new results in advanced micro-nano domains, properly new technologies under development and complementarity.

\section{CONCLUSIONS}

Intelligent Micro-Nano-Robotics is defined as a science of the future, for making micro-nano-devices, micro-nano-systems and micro-nano-products by employing micro-nano-materials.

Micro-Nano-intelligent robot learns from nature to make products and systems much smaller, much lighter, more durable, more efficient, all used in the interest and the good of man and planet.

Micro-Nano-Robotics Intelligent implications contribute ecological, social, ethical and medical.

\section{REFERENCES}

Arai, T., et al. (1995), Micro Manipulation Based on Micro Physics, Proc. Of. IEEE / RSJ, Int. Conf on Intelligent Robots and Systems (waste), pp. 236-241, Pittsburgh, Pennsylvania

Bartlett, P. N. (1996), Handbook of Chemical and Biological Sensors, 10P Publishing, Philadelphia

Fatikow, S., Rembold, U (1999), Microsystems Technology and Microrobotics, Technical Publishing

Gheorghe, Ion Gheorghe, Badita, Liliana (2009), Advanced Micro- and Nanotechnologies in Mechatronics, 978-60692267-1-1; Cefin Publishing House

Gheorghe, Ion Gheorghe (2009), Intelligent Micro-engineering, 978-606-92267-2-8 